\title{
Experimental and Numerical Study of Fatigue Damage Assessment under Combined High and Low Cycle Loading
}

\author{
Chaoshuai Han, ${ }^{1}$ Xianqiang Qu ${ }^{D},{ }^{1}$ Yongliang Ma $\left(\mathbb{D},{ }^{2}\right.$ and Dexin Shi ${ }^{1}$ \\ ${ }^{1}$ College of Shipbuilding Engineering, Harbin Engineering University, Harbin, Heilongjiang 150001, China \\ ${ }^{2}$ College of Shipping and Navy Architecture, Chongqing Jiaotong University, Chongqing, China \\ Correspondence should be addressed to Xianqiang Qu; quxianqiang@hrbeu.edu.cn
}

Received 31 January 2018; Accepted 17 April 2018; Published 12 July 2018

Academic Editor: Vadim V. Silberschmidt

Copyright (C) 2018 Chaoshuai Han et al. This is an open access article distributed under the Creative Commons Attribution License, which permits unrestricted use, distribution, and reproduction in any medium, provided the original work is properly cited.

\begin{abstract}
This paper studies fatigue damage under combined high and low cycle loading. The interaction of two types of loading will greatly reduce fatigue life of structures. Existing methods usually neglect the minor high cycle loading, which obviously underestimates the total fatigue damage. To solve this problem, four methods are proposed and extended for the case of combined loading. Numerical simulations based on the superposition of two sine waves are performed. Experiments are carried out using "DH36" smooth roundtype specimens. Damage predictions results by four methods are compared to the experimental results. It is concluded that some methods give satisfactory fatigue damage estimations and can be regarded as a safe design tool in engineering application.
\end{abstract}

\section{Introduction}

Many important engineering structures experience low cycle loading and are also subjected to high cycle loading [1]. A typical example may be a wave frequency response due to wave and a high frequency response excited by whipping/springing for some flexible and fast merchant ships and containerships [2-4]. During one voyage, wave frequency response includes low frequency major stress cycles which can be regarded as low cycle loading, while springing and whipping response produces high frequency minor stress cycles which is considered to be high cycle loading. Many studies show that fatigue damage induced by whipping/springing response takes up an important contribution to the total fatigue damage [2-4]. Mao finds that HF response produced by whipping/springing contributes fatigue damage of larger than 30\% although HF response induces average energy of less than 3\% [2]. Thus, it is necessary to predict fatigue damage under combined high and low cycle loading.

For the combination of low cycle loading and high cycle loading, a simple treatment is to ignore the high frequency minor cycle loading [5]. However, the coupling effect of LCL and HCL will greatly reduce fatigue life of structures when these high frequency minor stress cycles are superimposed on part of the low frequency major stress cycles. Therefore, the above approach is not suitable for safe design of structures. Another traditional method is to transform combined cycle loading into single cycle loading. After that, the amplitude of single cycle loading is the sum of low cycle loading and high cycle loading, and the cycle number is equal to the cycle number of low cycle loading. Unfortunately, several studies show that single cycle treatment cannot evaluate the fatigue damage due to high frequency minor stress cycles. The third method is the simple damage summation method; damage from high and low cycle loading is calculated independently and then simply added [6]. This method provides highly nonconservative damage predictions because of the nonlinear relation of fatigue damage and stress. Recently, some researchers reveal the interaction of LCL and HCL in terms of superposition of damage from the separate loading [7-10]. $\mathrm{Fu}$ and Cebon assumed the combination of two sine waves in the time domain [7]. By rainflow counting algorithm, the combined damage can be split up into two major parts: one is the large cycles; the other is the small cycles. The total damage can then be the sum of the large cycles and the small cycles. Based on the above idea, Lotsberg derived a formula associated with the individual frequency and fatigue damage, which has been introduced into the DNV rule for 
estimating the fatigue damage due to high and low cycle loading $[8,9]$. In addition, there are other methods which may be used in terms of combination of damage equivalent stress ranges. If nothing is known on the phase relation, an in-phase superposition of damage equivalent stress ranges from LCL and HCL can be combined fatigue loading; this treatment may provide a conservative combined fatigue damage prediction [11]. More recently, Han and Ma derived a simple formula which is expressed as a function of individual damage [12]; this method will be applied to calculate the combined fatigue damage induced by high and low cycle loading. In addition, for combination of low cycle fatigue and high cycle fatigue, Szala and Ligaj present a hybrid method to calculate construction steel fatigue life; this hybrid method points out that low cycle fatigue is performed with strain based approach and high cycle fatigue is computed through stress based approach; the total fatigue damage is summed with low cycle fatigue damage and high cycle fatigue damage [13]. The combined stress process may be a broad process; at this situation, Szala and Ligaj consider using some models of two-parameter fatigue characteristics (model IM [14], model II [14], model III [14], and model IV [14]) for determining the equivalent load spectra; based on this, fatigue life can be obtained in the preliminary stage of structural design process [15].

The purpose of this paper is to find a method to evaluate fatigue damage due under combined high and low cycle loading. Numerical simulations are first carried out to validate the accuracy of different methods. Then, experimental study is conducted and experimental results are compared with some proposed methods.

\section{Theory Background}

2.1. Basic Fatigue Theory. Fatigue life is based on $S$ - $N$ curves, which are obtained from fatigue tests of small specimens in test laboratories. For an $S-N$ curve, it can be expressed as

$$
N=K \cdot S^{-m}
$$

where $N$ is predicted number of cycles to failure for stress range and $S, K$, and $m$ are constants associated with the material properties, the ratio of stress, and the type of loading. In particular, it should be noted that the material parameter $m$ has a significant impact on calculated fatigue damage since the value of $m$ depends on different materials. For instance, $m$ is usually taken as 3, 4, 5 for welded steel, while it is taken as 8,9,10 for ceramics and glass and even 20 for smooth round bars. It has been proven that the theoretical results of fatigue prediction are different for $m=3$ and $m=4$ by Jiao and Moan [16]. In addition, it is convenient to assume $S$ - $N$ curve with one slope. In Kuhn's thesis, $m=4$ is used as a linear approximation of the bilinear curve of $m=3$ and $m=5$ [11]. In order to make the process of derivation brief and convenient, the influence of the mean stresses is negligible.

Figure 1 illustrates three curve types proposed by DNV rules [9]. Type A is a bilinear curve which has a transition in slope from $m=3$ to $m=5$, and type B is an only one slope

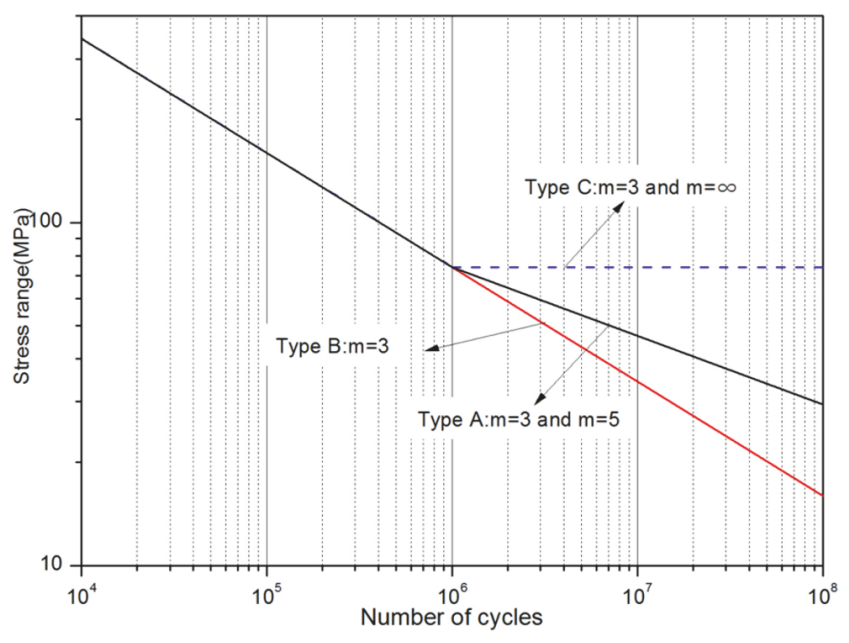

Figure 1: Example of $S-N$ curve according to DNV rule.

curve after the inverse slope of $m=3$ is extended, while type $\mathrm{C}$ is used when a fatigue limit is considered.

Note that, in most cases, type A with bilinear inverse slopes is the most appropriate for analyzing the fatigue damage on the certification level. However, in the preliminary design stage and when an analytical evaluation of combined fatigue damage is desired, the assumption of a constant slope is convenient. In this paper, numerical simulations, experimental study, and derivation of combined damage formula are based on a one-slope $S-N$ curve.

Fatigue deterioration or damage, $D$, is defined as the ratio between the reference number of cycles during the design lifetime and predicted number of cycles to failure. The total damage is calculated under the assumption of linear cumulative damage, i.e., Palmgren-Miner rule [17], which equals the summation of the ratio between the reference number of cycles, $n_{i}$, and the predicted number of cycles, $N_{i}$, for all stress range classes.

$$
D=\sum_{i=1} \frac{n_{i}}{N_{i}} .
$$

In order to achieve all stress ranges, the rainflow cycle counting originally proposed by Matsuishi and Endo is selected as the only counting procedure [18, 19], and the approach has been widely adopted and considered to be the most efficient counting method.

2.2. Damage Equivalent Theory. In general, it is convenient to introduce damage equivalent, constant amplitude stress range, which can be regarded as a concise description of fatigue damage. It should be noted that the principle of equal damage must be followed. For a constant slope $S-N$ curve, equivalent stress range depends only on the slope parameter $m$, the material parameter $K$, the range of cycles, and an arbitrary reference number of cycles. For example, the damage with the number of stress cycles, $k$, in a stress time history can be equivalent to the damage with only one 
stress cycle with respect to the reference number of cycles, $n_{r}$, namely, by

$$
\sum_{i=1}^{k} \frac{n_{i}}{N_{i}}=\frac{n_{r}}{N_{\Delta \sigma_{\mathrm{eq}}}}
$$

The equivalent stress range can be then obtained by the union of (2) and (3) as

$$
\Delta \sigma_{\mathrm{eq}}=\left[\frac{1}{n_{r}} \cdot \sum_{i=1}^{k}\left(n_{i} \cdot \Delta \sigma_{i}^{m}\right)\right]^{1 / m},
$$

where $N_{\Delta \sigma_{\text {eq }}}$ denotes the number of cycles to failure for the equivalent stress range, $\Delta \sigma_{\text {eq }}$, and $\Delta \sigma_{i}$ is the stress range for class $i$.

\section{Damage Superposition Methods during the Fatigue Analysis}

3.1. Arithmetic Superposition of Stress Range. A simplistic formula is proposed for calculating the combined fatigue loading which is based on the arithmetic summation superposition of the equivalent stress range.

$$
\Delta \sigma_{\mathrm{eq}}=\Delta \sigma_{L}+\Delta \sigma_{H}
$$

Under combined low cycle and high cycle loading, low cycle loading induced fatigue damage $D_{L}$ and high cycle loading induced fatigue damage $D_{H}$ can be calculated, separately, according to (1) and (2):

$$
\begin{aligned}
D_{L} & =\frac{\left(\Delta \sigma_{L}\right)^{m} n_{L}}{K} \\
D_{H} & =\frac{\left(\Delta \sigma_{H}\right)^{m} n_{H}}{K}
\end{aligned}
$$

With respect to damage equivalent theory, $D_{L}$ can be equivalent to the damage produced by a stress range of $\Delta \sigma_{\mathrm{eq}, H}$ and the number of cycles $n_{H}$.

$$
D_{L}=\frac{\left(\Delta \sigma_{L}\right)^{m} n_{L}}{K}=\frac{\left(\Delta \sigma_{\mathrm{eq}, H}\right)^{m} n_{H}}{K}
$$

$\Delta \sigma_{\mathrm{eq}, H}$ can then be derived as

$$
\Delta \sigma_{\mathrm{eq}, H}=\Delta \sigma_{L} \cdot\left(\frac{n_{L}}{n_{H}}\right)^{1 / m}
$$

According to (5), the equivalent stress range of combined cycle loading can be obtained:

$$
\Delta \sigma_{\mathrm{eq}, L H}=\Delta \sigma_{\mathrm{eq}, H}+\Delta \sigma_{H}
$$

By incorporating (1), (2), (6), and (7) with (10), a combined damage formula can be obtained:

$$
D_{c}=\left(D_{L}^{1 / m}+D_{H}^{1 / m}\right)^{m}
$$

3.2. Quadratic Superposition of Stress Range. Another superposition method is based on the quadratic superposition of the equivalent stress range. The formula is as follows:

$$
\Delta \sigma_{\mathrm{eq}, L H}=\sqrt{\Delta \sigma_{\mathrm{eq}, L}^{2}+\Delta \sigma_{\mathrm{eq}, H}^{2}}
$$

According to a similar process with (11), another combined damage formula can be obtained:

$$
D_{c}=\left(D_{L}^{2 / m}+D_{H}^{2 / m}\right)^{m / 2}
$$

Equation (13) will be used to calculate the combined fatigue damage due to low cycle loading and high cycle loading.

3.3. DNV Method. Fatigue damage is increased by assuming that the high cycle loading can be added to that of the low cycle loading to get a stress range $\left(\Delta \sigma_{L}+\Delta \sigma_{H}\right)$ and the number of cycles $n_{L}$. Then fatigue damage from the high cycle loading can be increased with a stress range $\Delta \sigma_{H}$ and the number of cycles $\left(n_{H}-n_{L}\right)$. Hence, the total fatigue damage as DNV method can be obtained as follows:

$$
D_{\mathrm{DNV}}=\frac{\left(\Delta \sigma_{L}+\Delta \sigma_{H}\right)^{m}}{K} \cdot n_{L}+\frac{\left(\Delta \sigma_{H}\right)^{m}}{K}\left(n_{H}-n_{L}\right),
$$

where $\Delta \sigma_{L}$ is the stress range of low cycle loading, $n_{L}$ is the number of cycles of low cycle loading, $\Delta \sigma_{H}$ is the stress range of high cycle loading, and $n_{H}$ is the number of cycles of high cycle loading.

3.4. Quadratic Superposition of Fatigue Damage. Temple depicted an approach of the combined fatigue damage based on the quadratic superposition of the separate fatigue contributions, which has been applied to the calculation of fatigue damage in the Utgrunden offshore wind farm [20].

$$
D_{\text {Temple }}=\sqrt{D_{L}^{2}+D_{H}^{2}}
$$

\section{Numerical Validation}

Trapezoidal wave is generally used to replace the low cycle loading in experimental study and numerical simulations. However, trapezoidal wave is not consistent with the actual loading. Relatively, sine wave is close to the practical situation and is thus adopted in this paper. Simplified HCL and LCL can be seen in Figure 2. Assuming that $\Delta \sigma_{H}$ and $\Delta \sigma_{L}$ are the stress range of two sine waves, $f_{H}$ and $f_{L}$ are the individual frequency. Two new parameters are defined as follows:

$$
\begin{aligned}
& R_{\sigma}=\frac{\Delta \sigma_{H}}{\Delta \sigma_{L}} \\
& R_{f}=\frac{f_{H}}{f_{L}}
\end{aligned}
$$

The ratio of loading $R_{\sigma}$ and the ratio of frequency $R_{f}$, as well as $S$ - $N$ curve parameter $m$, are considered as the main 


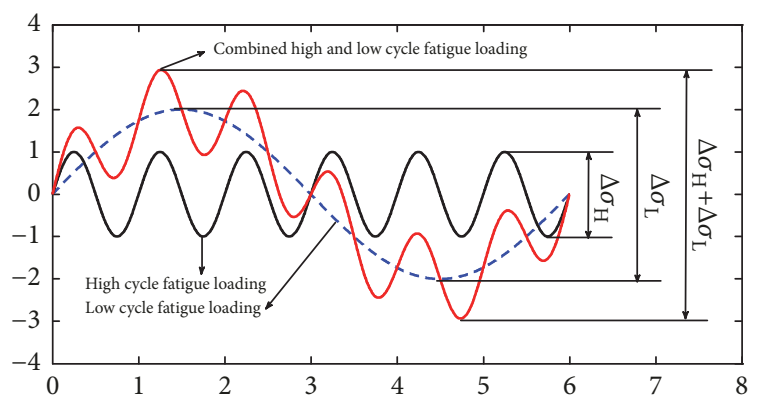

Figure 2: Schematic view of LCL, HCL, and combined cycle loading.

factor which influences the fatigue damage obtained from previously mentioned methods. In numerical simulations, the value of $R_{\sigma}$ is $4: 1,3: 1,2: 1,1: 1,1: 2,1: 3$, and $1: 4$. The value of $R_{f}$ is $10,50,100$, and $300 . m$ is defined from 1 to 20 with an increment of 1 .

Time-domain rainflow damage in a sufficiently long time series can be used as a reference solution and is compared with estimations by arithmetic superposition of stress range method, quadratic superposition of stress range method, DNV method, and quadratic superposition of fatigue method. Presented results in all figures are given in terms of the fatigue damage ratio which is defined in (17):

$$
\rho=\frac{\bar{D}}{D_{\mathrm{RFC}}},
$$

where $D_{\mathrm{RFC}}$ is the fatigue damage from numerical simulations; it can be calculated through rainflow cycle counting for combined stress time series, associating with $S$ - $N$ curve and Palmgren-Miner rule. $\bar{D}$ is the theoretical damage estimate computed by each of the methods previously proposed. It is defined that when $\rho>1$, the fatigue damage estimation is conservative. On the contrary, when $\rho<1$, the fatigue damage estimation is not conservative.

Figure 3 plots the damage estimation by the arithmetic summation of equivalent stress range. When $R_{\sigma}>1$, the factor $\rho$ increases with the increasing of $m$; when $R_{\sigma} \leq 1$, the factor $\rho$ firstly increases and then tends to steadiness with the increasing of $m$. Keeping $m$ and $R_{f}$ fixed, the factor $\rho$ decreases with the decreasing of $R_{\sigma}$. In addition, by comparison with Figures 3(a), 3(b), 3(c), and 3(d), the factor $\rho$ is raised with the increasing of $R_{f}$. Overall, when $m$ is equal to 3,4 , and 5 or the value of $R_{\sigma}$ and $R_{f}$ is very low, this method provides reasonably conservative fatigue damage prediction.

Figure 4 shows the damage estimation by DNV method. The value of $R_{f}$ almost has no effect on the factor $\rho$. Only when $R_{\sigma}<1$ and $m<6$, the factor $\rho$ is a little higher. In other cases, very good agreement between DNV method and numerical simulations can be observed.

Figure 5 exhibits the damage estimation by quadratic superposition of stress range method. In most cases, the method highly underestimates the combined fatigue damage. Only when $m \leq 5$, the method can give an accurate fatigue damage estimation.

Similarly to quadratic superposition of stress range method, quadratic superposition of fatigue damage method can provide good damage results only in a small range. In other cases, it cannot give precise damage evaluation (as seen in Figure 6).

\section{Experimental and Numerical Validation of Superposition Approaches}

\subsection{Experimental Validation Based on Smooth Round-Type Specimens}

5.1.1. Test Setup. A high strength marine structural steel named DH36 is used in this paper, which is widely used for constructions of ships and marine structures. The design of specimens follows the American Society for Testing and Materials (ASTM) [21]. Figure 7 exhibits size of smooth round-type specimens and the machined specimens, respectively. The left side in Figure 8 is the electrohydrautic servo fatigue test machine; the right side is the control interface.

5.1.2. Test of S-N Curve. According to test requirements of $S$ $N$ curve, complete rupture of specimens is defined as failure criterion. Six-grade stress levels are adopted in the test, and for every grade stress level one specimen is used to conduct a fatigue test until the failure criterion is attained. If the test data is abnormal, repeated test should be carried out for another specimen. The load is a symmetrical sine wave with a mean amplitude of zero. During the test, the value of loads and the number of cycles to failure for each specimen are recorded. The experimental data were then fitted using the least squares method as shown in Figure 9, with a variance of $97.3644 \%$, which proved that the reliability of the test data, and the fitted $S-N$ curve expression is as follows:

$$
\lg N=54.27057563816-19.83042291007 * \lg S
$$

From the above equation, two important parameters related to calculation of fatigue damage can be implied, namely, by $m=19.83042291007, \lg K=54.27057563816$.

5.1.3. Grouping of Test Load. As previously mentioned, the ratio of loads and the ratio of frequencies are the main factors which have a significant effect on fatigue damage. Based on that, five groups of loads are selected for experimental test, as shown in Table 1, and the first three groups (groups 13) represent the combination of low frequency plus small loads and high frequency plus large loads; the last two groups (groups 4-5) denote the combination of low frequency plus large loads and high frequency plus small loads.

Note that the ratio of loads is equal to that of stress ranges considering the linear relationship between loads and stress for test specimens,

$$
S=\frac{F}{\pi \cdot r^{2}}
$$

where $r$ is the diameter of specimens in the beeline part.

The fact that fatigue life has no concern with the loading path is widely accepted; thus triangle waves are adopted during the test instead of sine waves after the condition that 


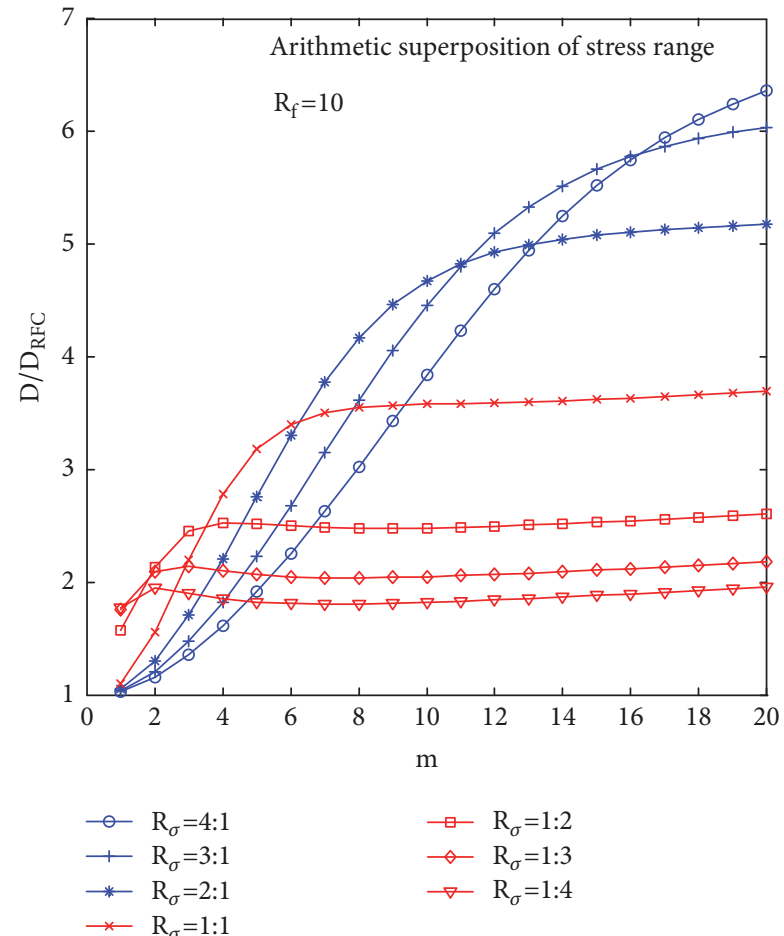

(a)

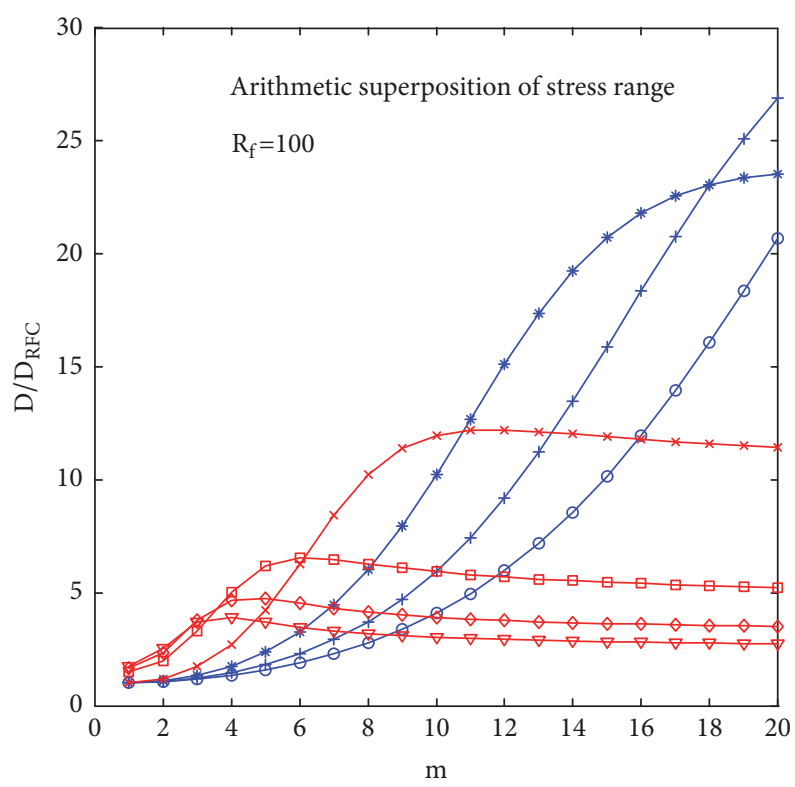

$$
\begin{array}{ll}
\multimap & \mathrm{R}_{\sigma}=4: 1 \\
+ & \mathrm{R}_{\sigma}=3: 1 \\
\text { * } & \mathrm{R}_{\sigma}=2: 1 \\
\text { — } & \mathrm{R}_{\sigma}=1: 1
\end{array}
$$

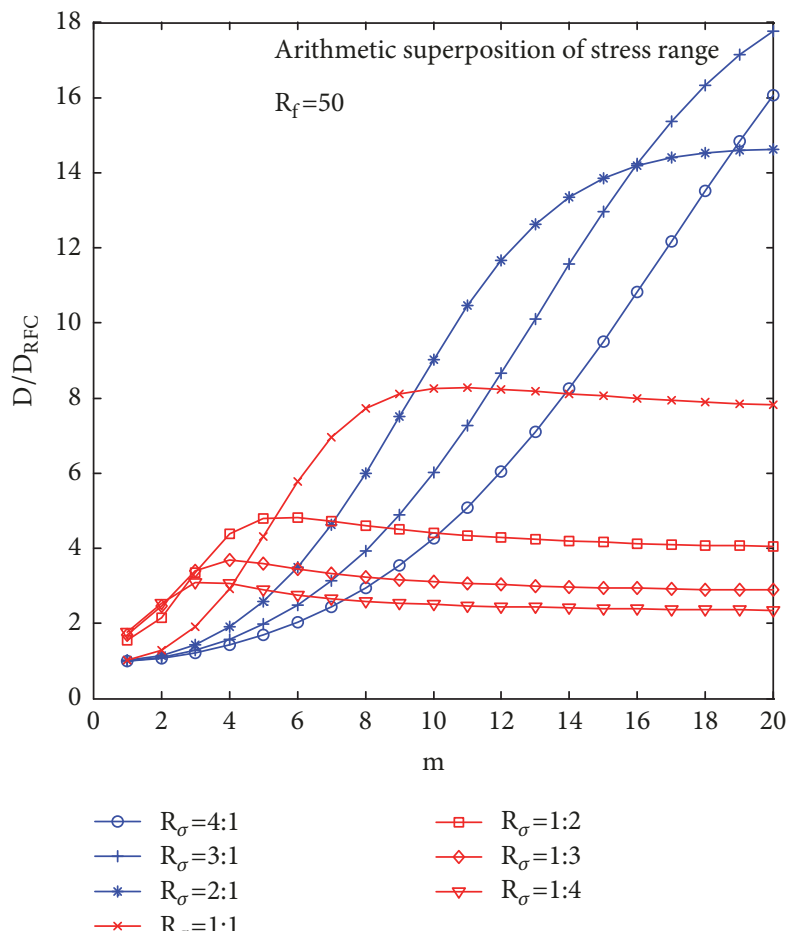

(b)

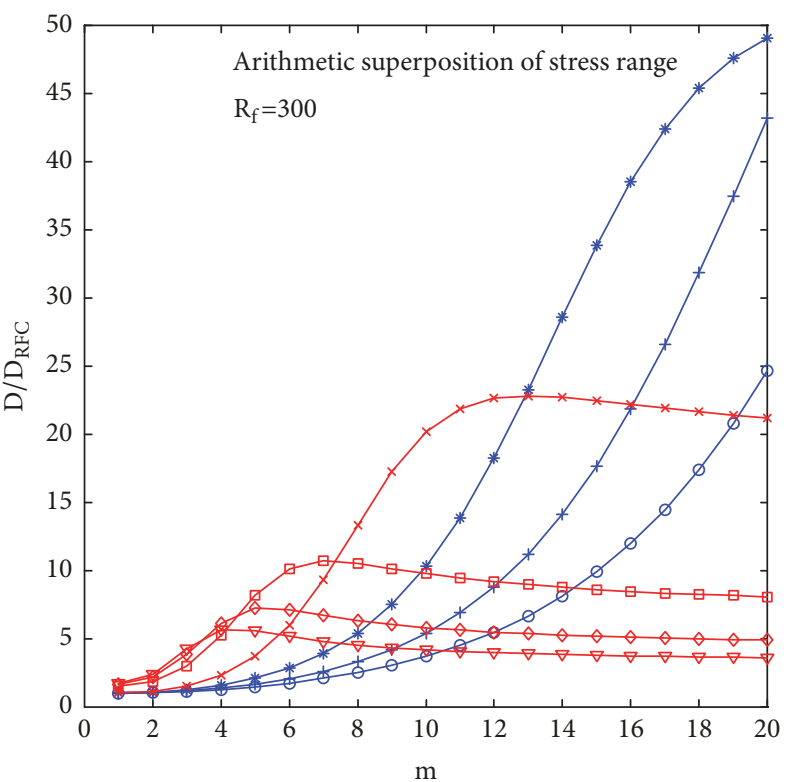

$$
\begin{array}{ll}
\multimap & \mathrm{R}_{\sigma}=4: 1 \\
+ & \mathrm{R}_{\sigma}=3: 1 \\
* & \mathrm{R}_{\sigma}=2: 1 \\
\rightarrow & \mathrm{R}_{\sigma}=1: 1
\end{array}
$$

(c)

(d)

FIGURE 3: Damage estimation by the arithmetic summation of equivalent stress range in the case of (a) $R_{f}=10$, (b) $R_{f}=50$, (c) $R_{f}=100$, and (d) $R_{f}=300$. 


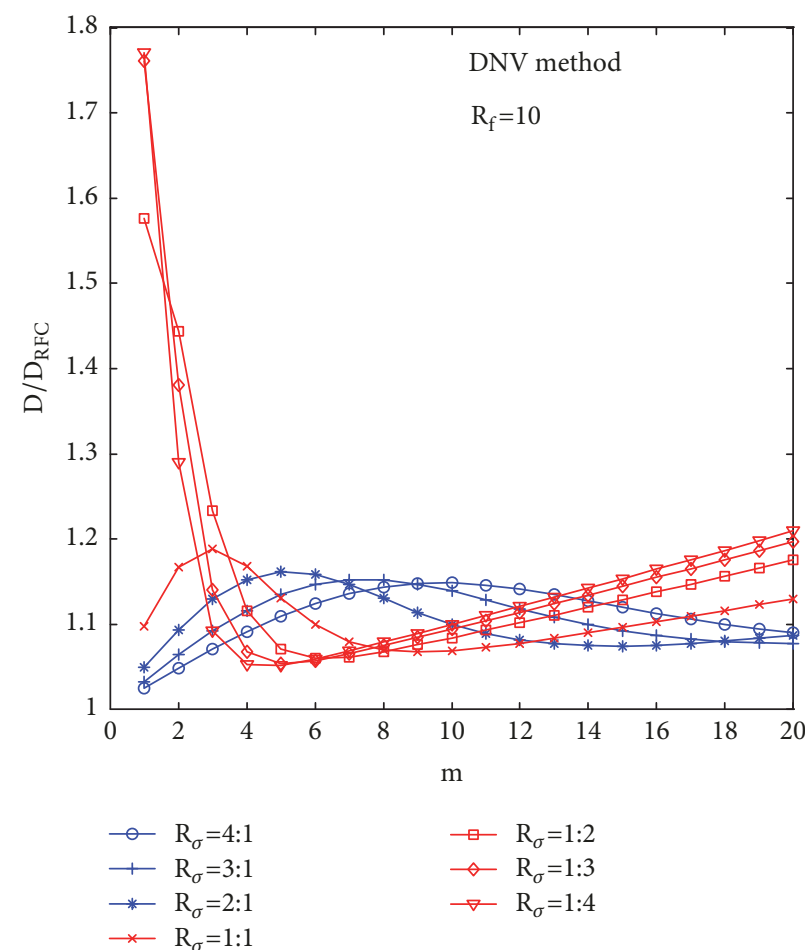

(a)

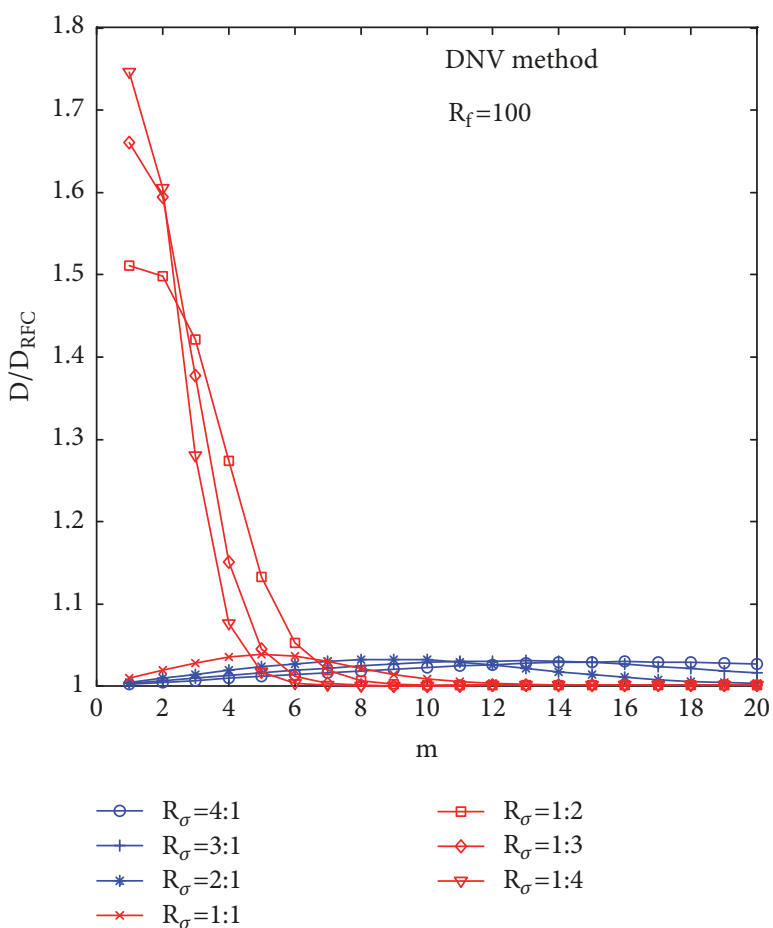

(c)

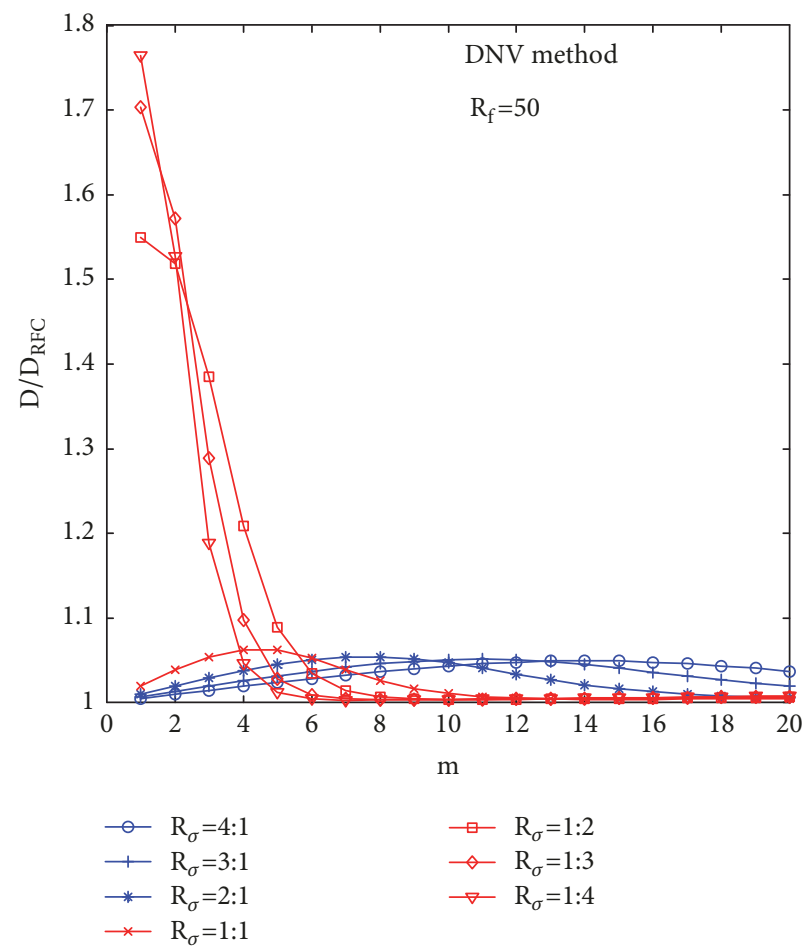

(b)

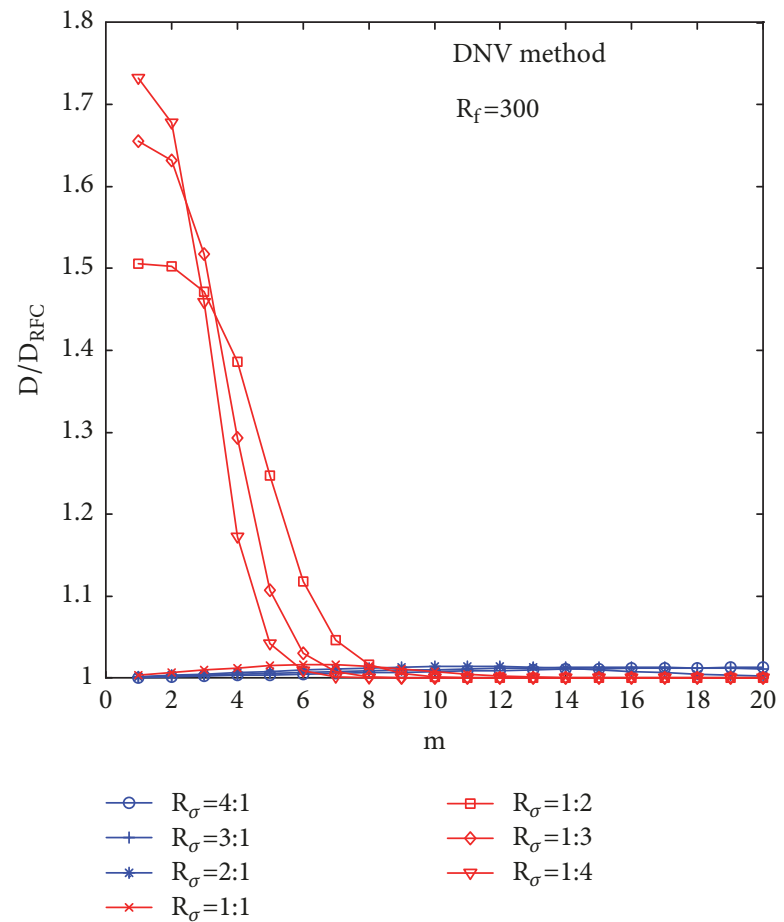

(d)

FIGURE 4: Damage estimation by DNV method in the case of (a) $R_{f}=10$, (b) $R_{f}=50$, (c) $R_{f}=100$, and (d) $R_{f}=300$.

they have the same peak and trough is fulfilled. The combined loading process can be seen as in Figure 10.

Different combinations have quite distinct time-domain characteristics, as seen in Figure 11.
5.2. Discussion of Experimental Results. For five load combinations, the number of test specimens is $4,4,5,4$, and 1 , respectively. The parts of ruptured test specimens after fatigue test are illustrated in Figure 12. The number of cycles to failure 


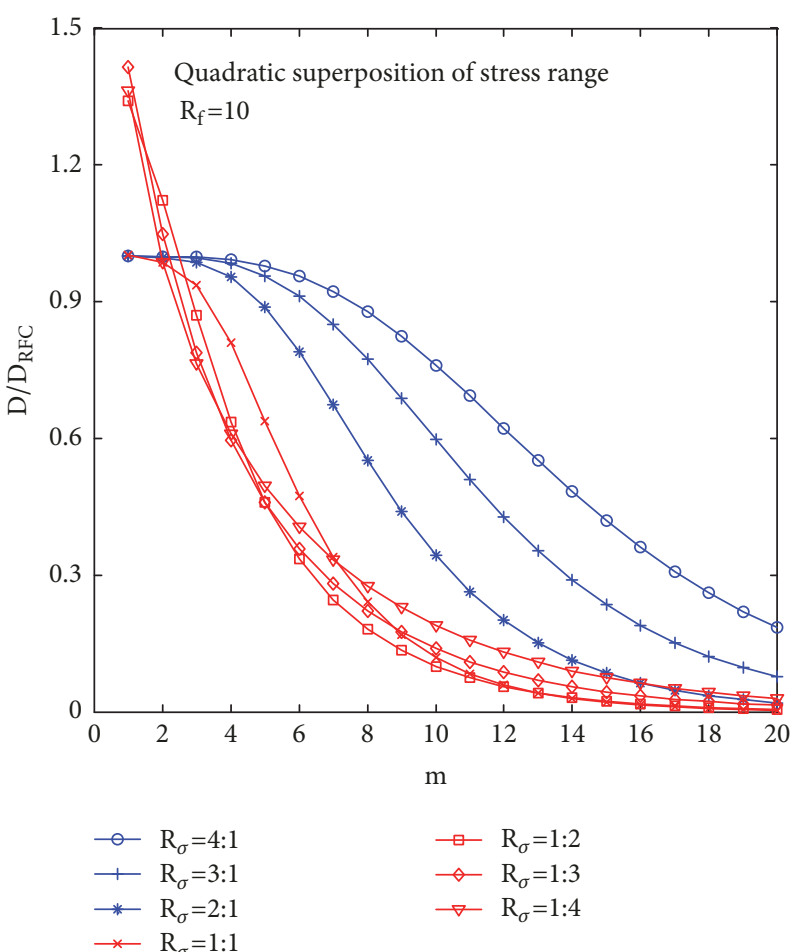

(a)

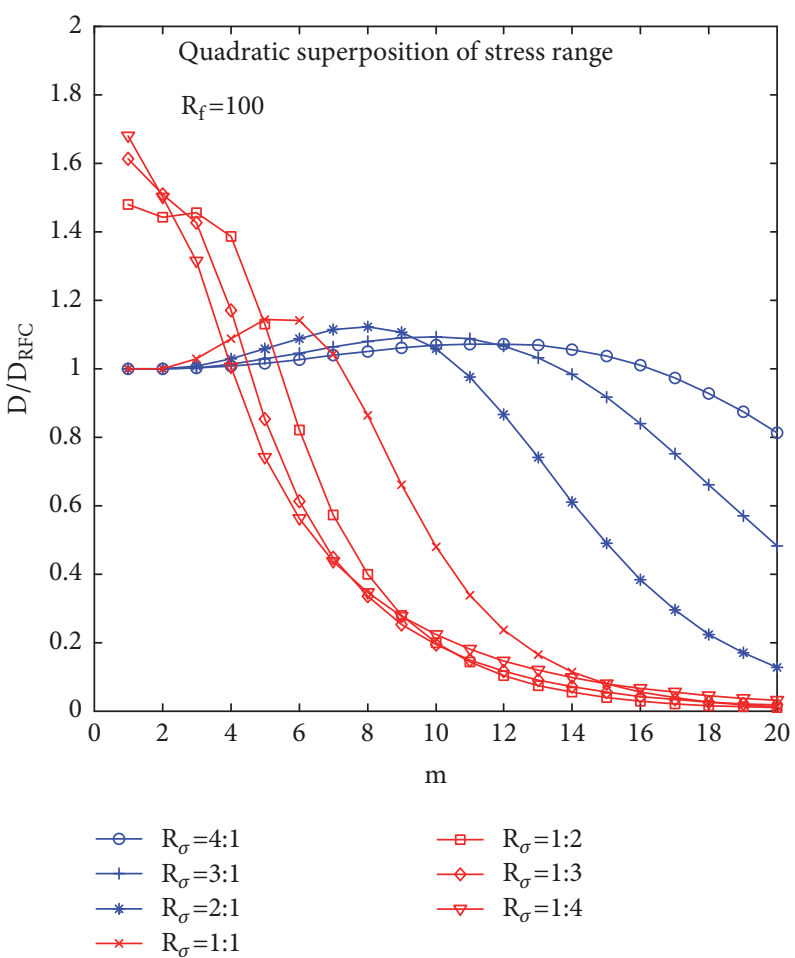

(c)

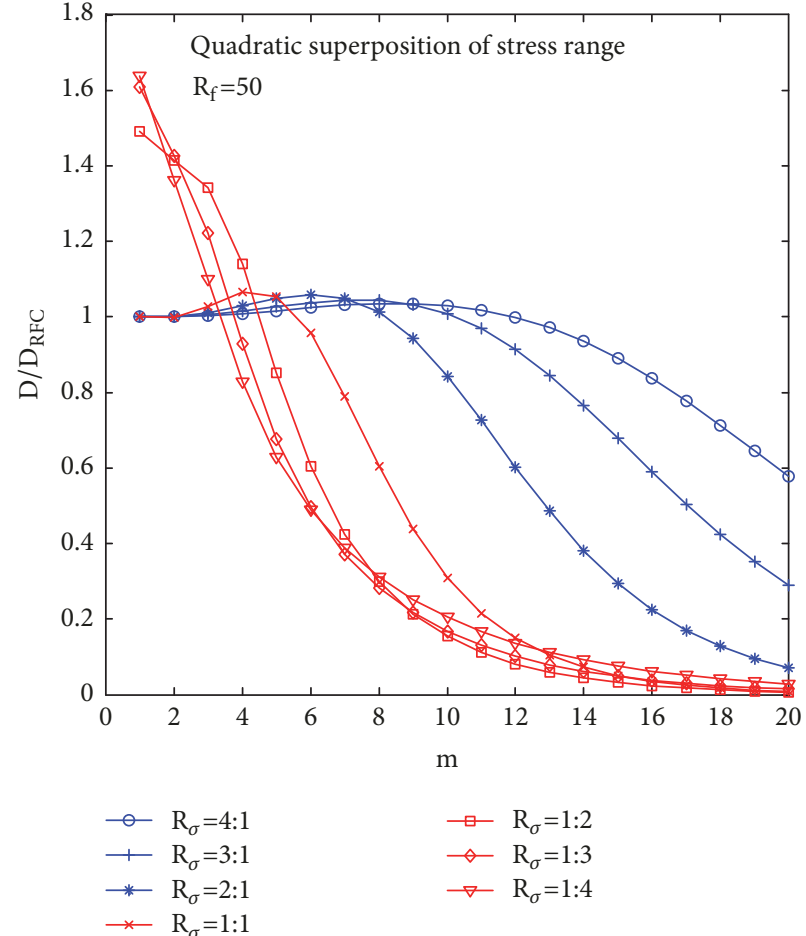

(b)

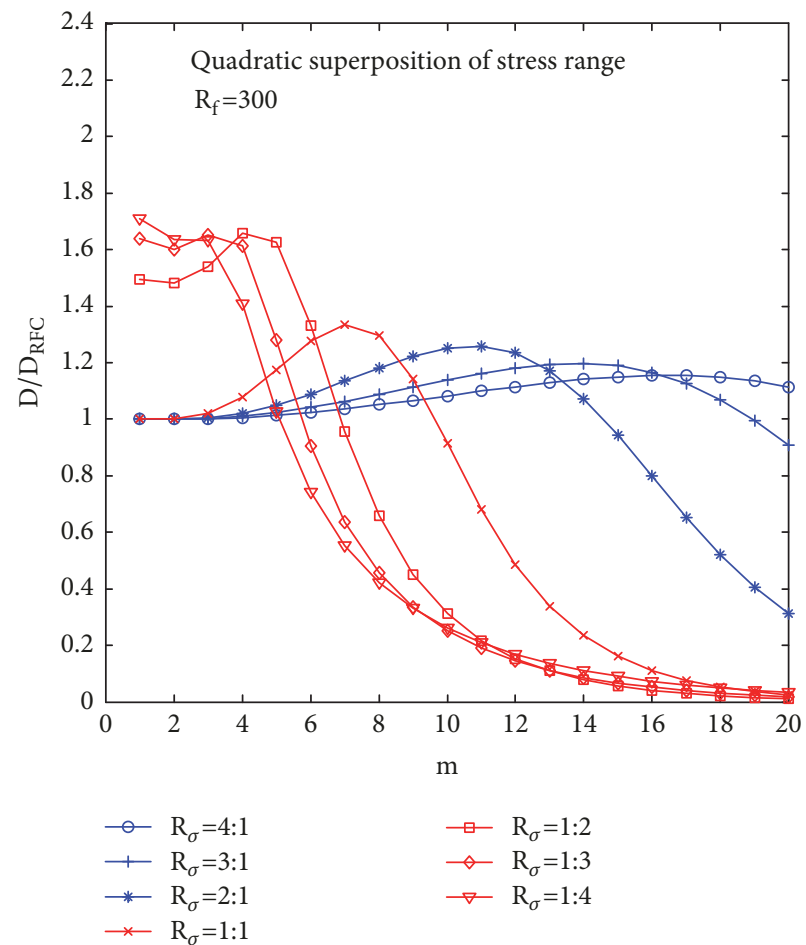

(d)

FIGURE 5: Damage estimation by direct quadratic superposition of equivalent stress ranges method in the case of (a) $R_{f}=10$, (b) $R_{f}=50$, (c) $R_{f}=100$, and (d) $R_{f}=300$. 


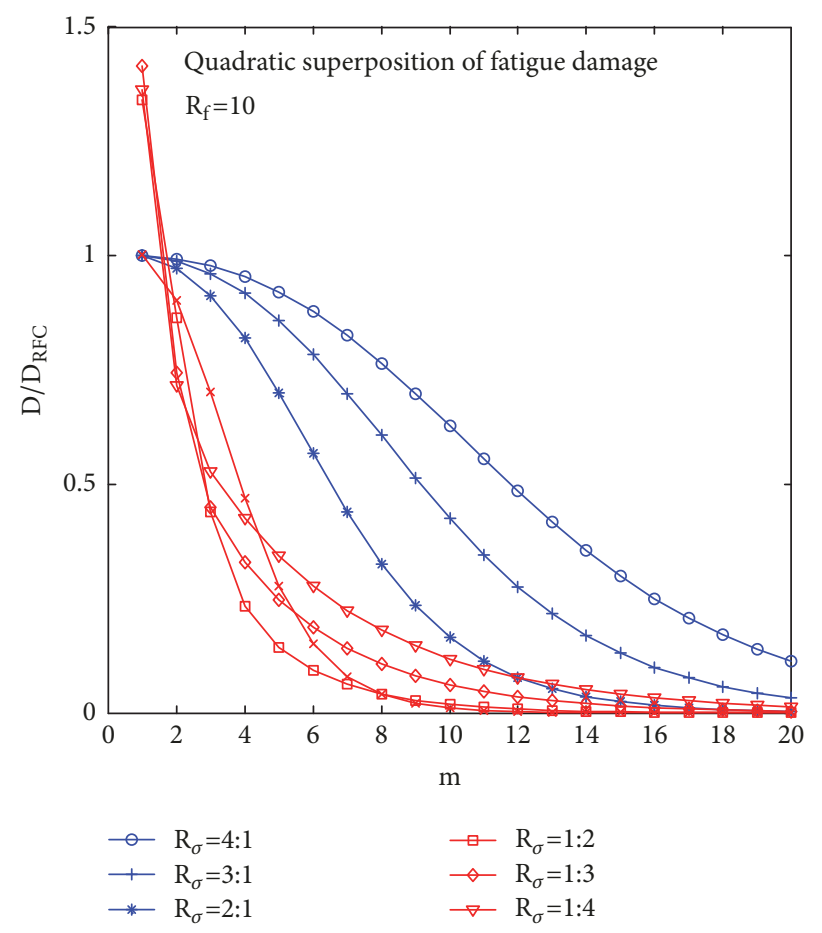

(a)

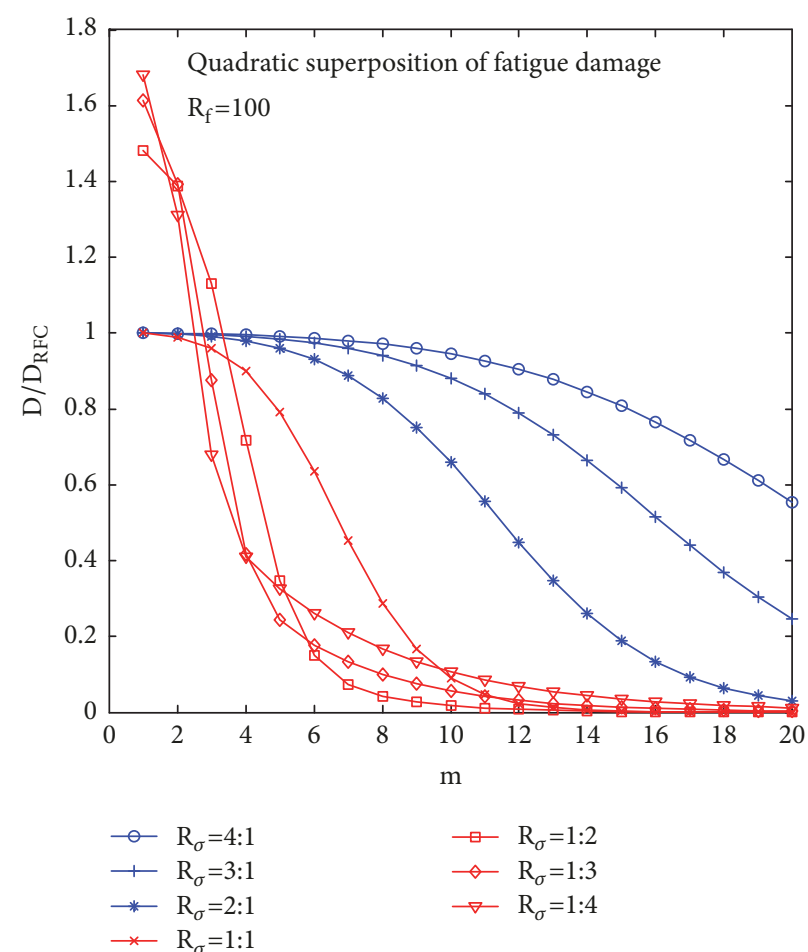

(c)

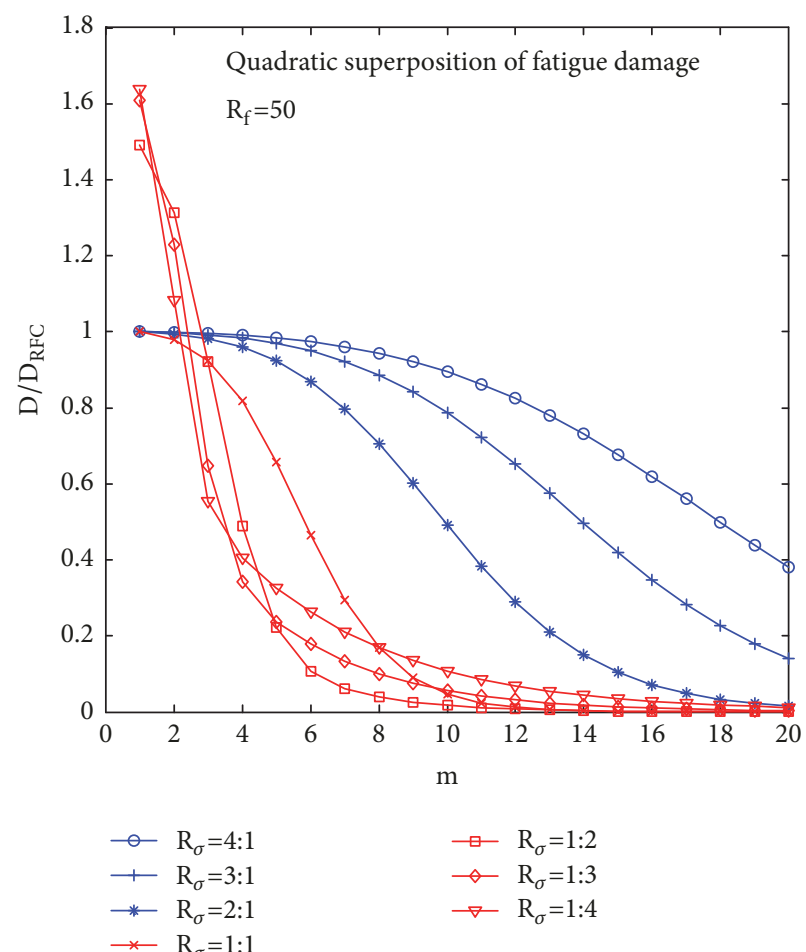

(b)

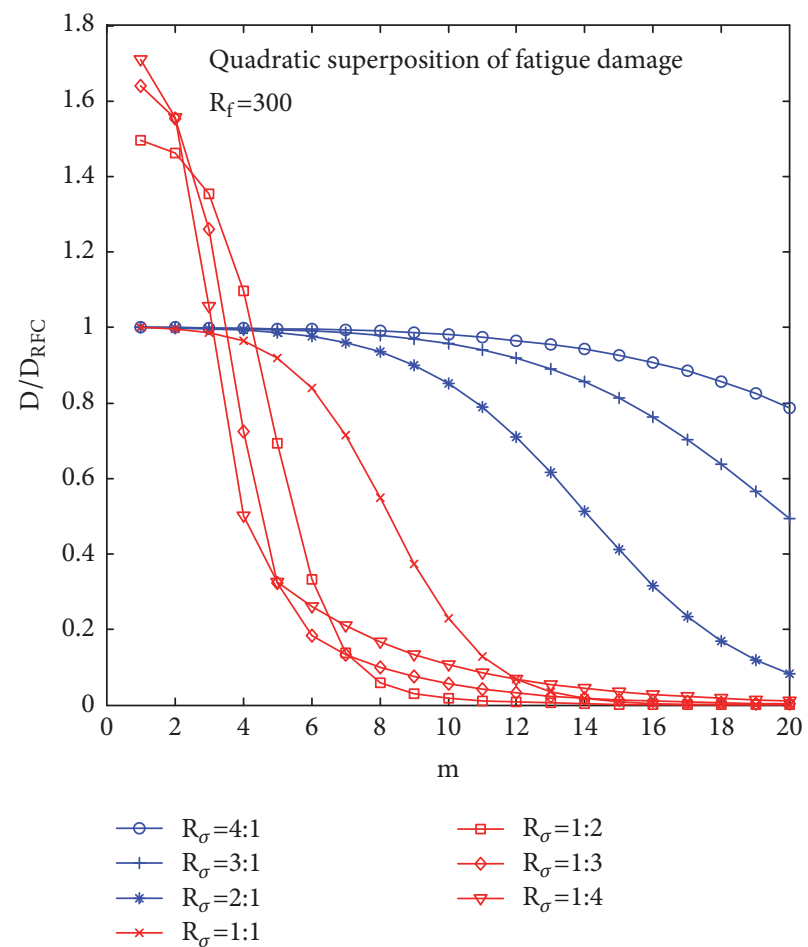

(d)

FIGURE 6: Damage estimation by Temple method in the case of (a) $R_{f}=10$, (b) $R_{f}=50$, (c) $R_{f}=100$, and (d) $R_{f}=300$. 
TABLE 1: Group of loads.

\begin{tabular}{lccccc}
\hline Group & load & Amplitude/KN & Ratio of loads & Frequency & Ratio of frequencies \\
\hline \multirow{2}{*}{1} & $F_{1}$ & 5.498 & \multirow{2}{*}{3.857} & 0.125 & 0.333 \\
& $F_{2}$ & 21.206 & \multirow{2}{*}{3.857} & 0.125 & 0.143 \\
\hline \multirow{2}{*}{3} & $F_{1}$ & 5.498 & \multirow{2}{*}{3.857} & 0.0625 & 1.143 \\
\hline \multirow{2}{*}{4} & $F_{2}$ & 21.206 & \multirow{2}{*}{0.259} & 0.125 & \multirow{2}{*}{16} \\
\hline \multirow{2}{*}{5} & $F_{1}$ & 5.498 & 0.143 & \multirow{2}{*}{1.143} \\
& $F_{2}$ & 21.206 & 0.259 & 0.0625 & 1 \\
\hline
\end{tabular}

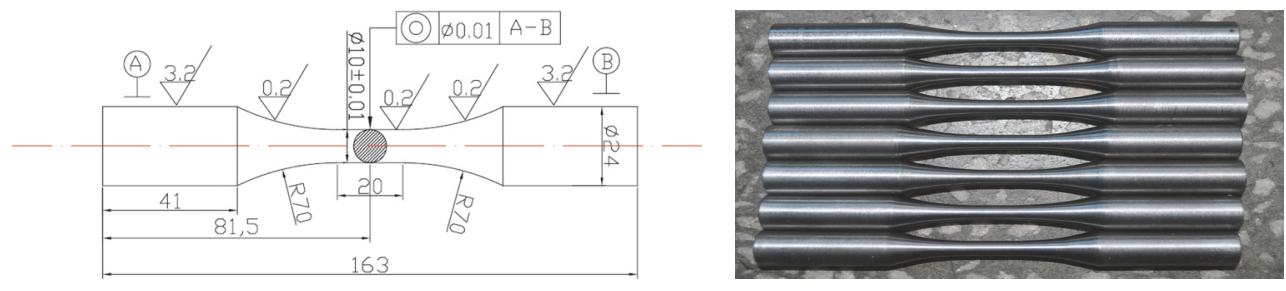

FIGURE 7: Designed specimens.

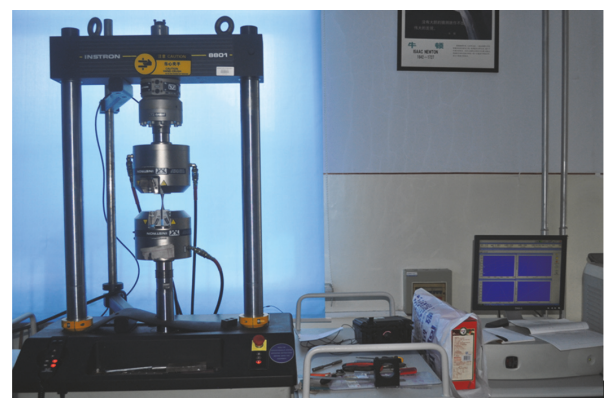

FIGURE 8: Test setup.

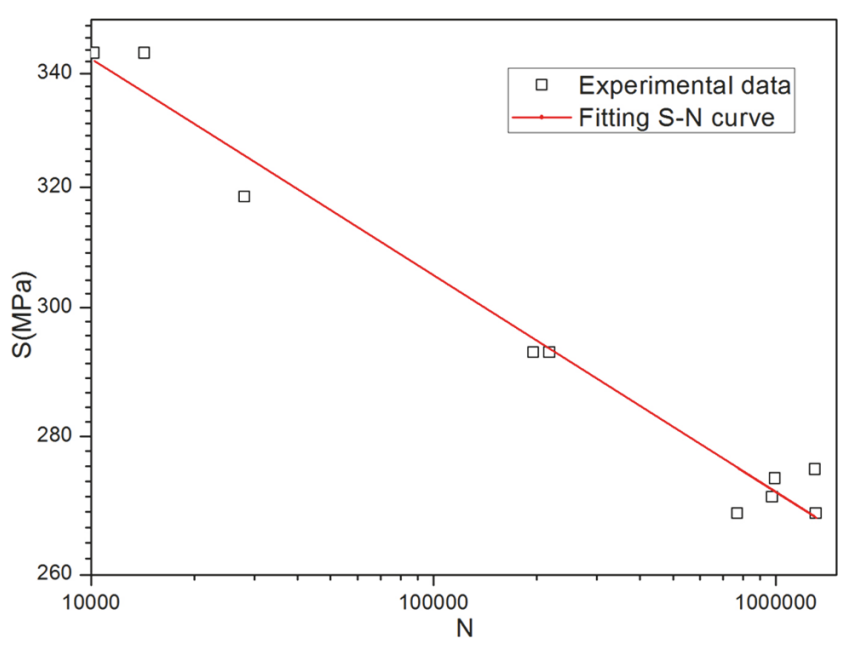

Figure 9: Test data of $S-N$ curve.

for each test specimen is recorded and then transformed into fatigue damage plotted in Figure 13. What is more, fatigue damage is calculated by the numerical simulations and some superposition formulae, based on the result of $S-N$ curve test. After that, the overall accuracy of all methods was studied.

First of all, fatigue damage based on time-domain simulations with rainflow counting is compared with results provided by the experiment. As can be seen in Figure 13, time-domain rainflow damage based on a one slope $S-N$ curve is basically the same as the results obtained from the experiment in most cases. However, in the case of $R_{\sigma}=3.857$, $R_{f}=16$, time-domain rainflow results are visibly lower than the experimental results for five test specimens in group 3. By this token, we infer that fatigue damage estimation from numerical simulations in this situation is inexact.

In addition, four superposition approaches including arithmetic superposition of stress range method, quadratic superposition of stress range method, DNV method, and quadratic superposition of fatigue damage method are compared with numerical results as seen in Figure 13.

Arithmetic superposition of stress range method can always provide highly conservative fatigue damage estimation. DNV method also gives conservative fatigue damage 

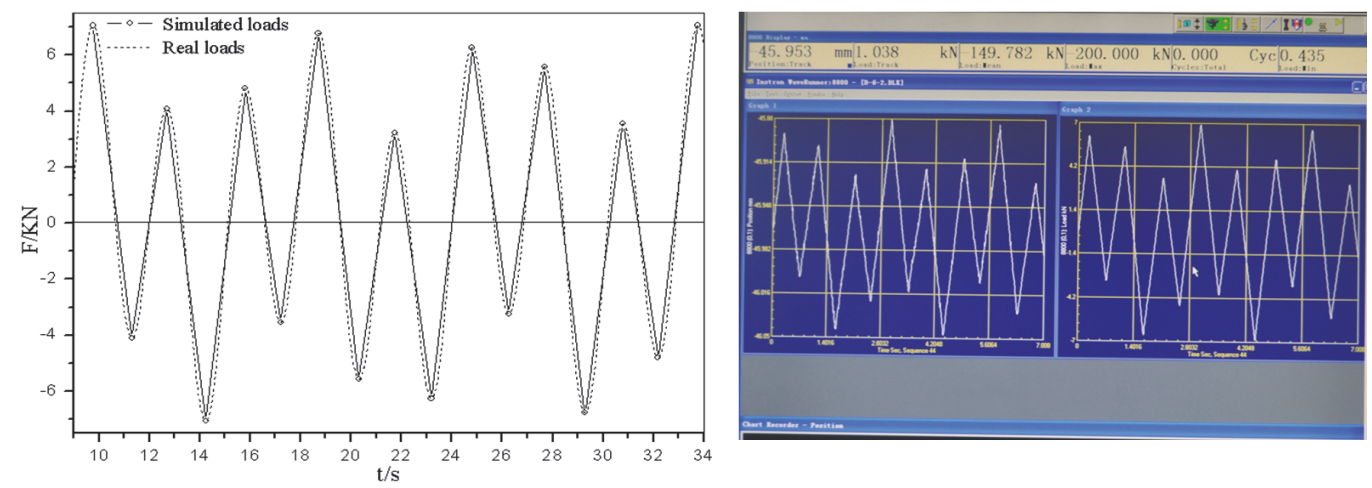

FIGURE 10: Detailed loading of combined process.
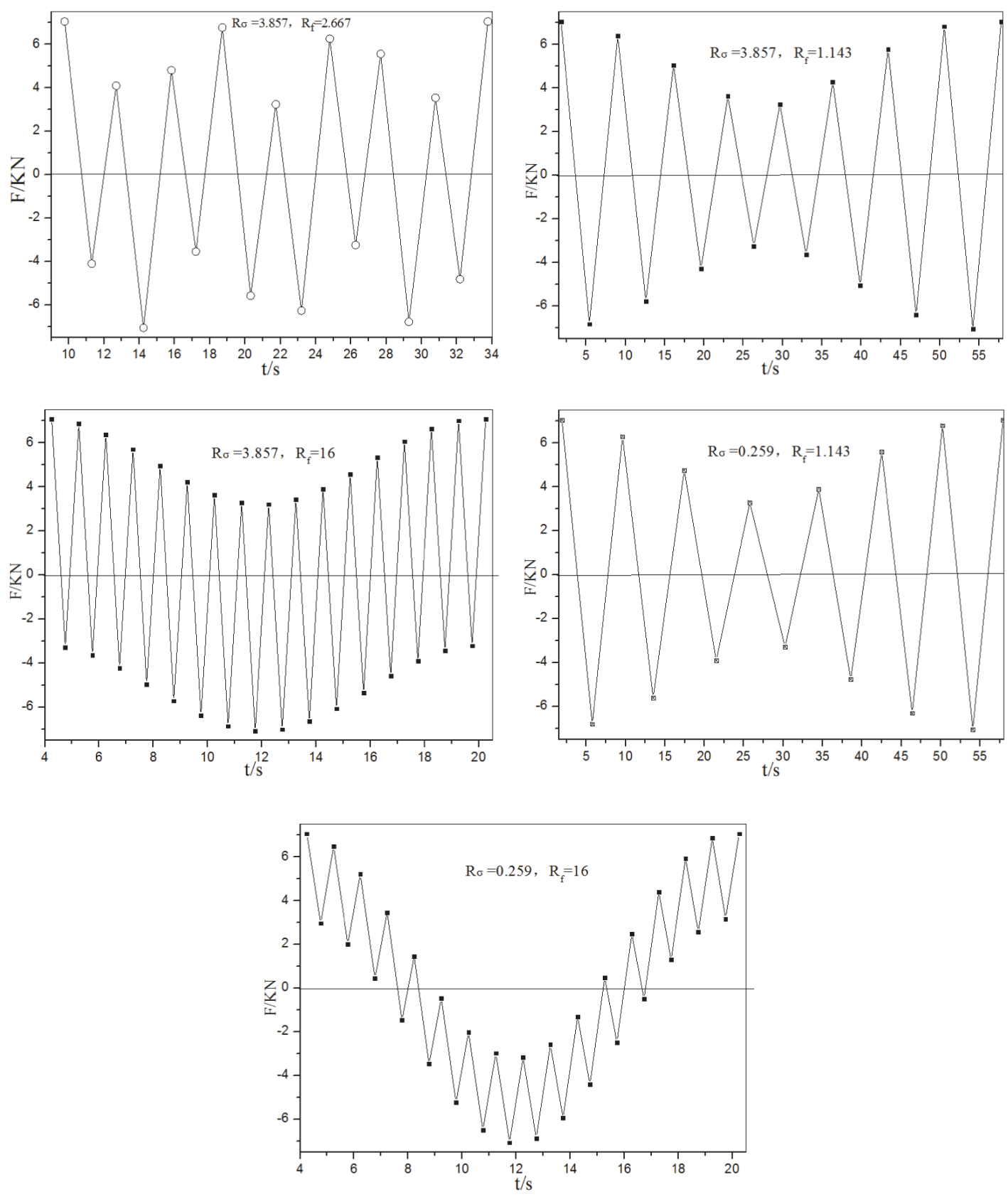

FIGURE 11: Simulated time history of combined process for experimental cases. 


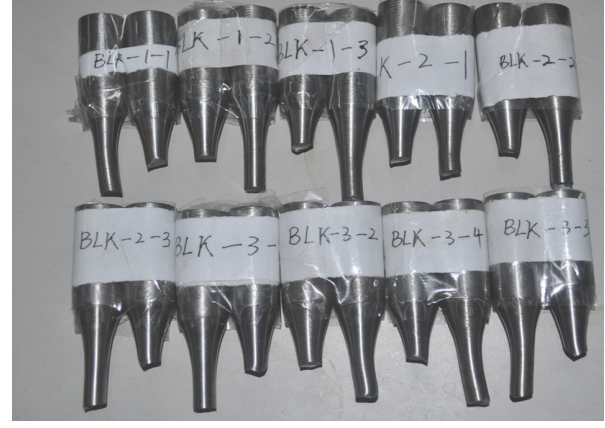

FIGURE 12: Ruptured specimens.

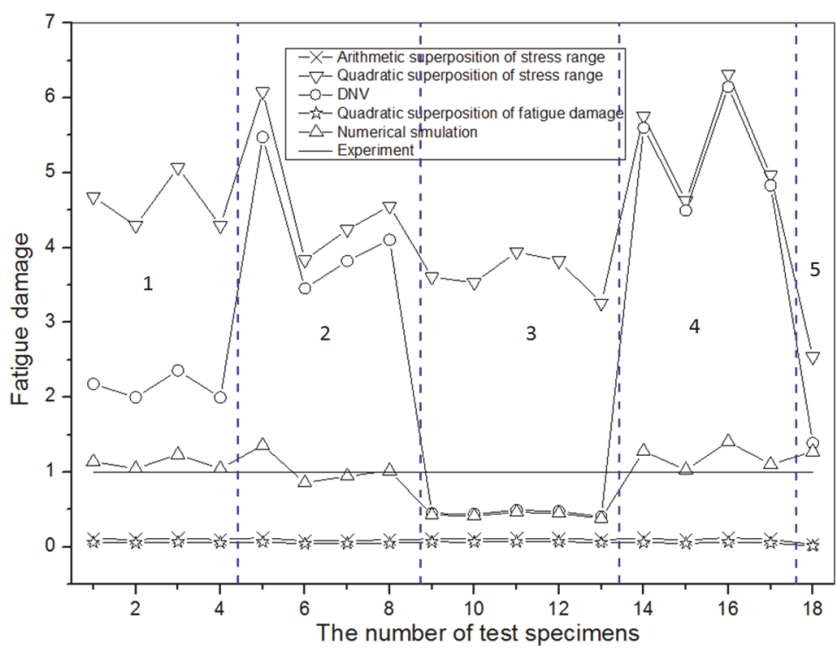

FIGURE 13: Fatigue damage result for different approaches.

prediction in four test load cases, but nonconservative in the third load case.

Quadratic superposition of stress range method and quadratic superposition of fatigue damage method give quite close fatigue damage results. However, both exhibit a tendency for a highly nonconservative results whatever the value of $R_{\sigma}$ and $R_{n}$ is, and the clear error of approximate to $-100 \%$ is unfavorable to estimation of the combined fatigue damage.

\section{Conclusions}

This paper studied the fatigue damage under combined high cycle and low cycle loading. Several methods are proposed for considering the interaction of high cycle and low cycle loading. The accuracy of new methods is validated with numerical simulations and experimental test. Some conclusions are as follows:

(1) Quadratic superposition of stress range method and DNV method can provide good fatigue damage results that are very close to simulated rainflow damage with a maximum error of about $20 \%$ when $1 \leq m \leq 5$ and $R_{\sigma}>1$.
(2) When $S$ - $N$ curve parameter $m$ is larger, four superposition methods provide unsatisfactory fatigue damage estimation with a maximum negative error of $100 \%$ when $m=20$.

(3) Fatigue predictions from numerical simulations produce negative 50\% compared with experimental results in some cases. In future work, experimental study on welded structures should be carried out to obtain typical $S$ - $N$ curve parameter $m$ (usually $m=3$, 4 , and 5) in ship and offshore structures.

(4) Overall, fatigue damage estimation error from different superposition methods increases with the increasing of the load ratio, the frequency ratio, and $S-N$ curve parameter.

\section{Data Availability}

The data used to support the findings of this study are available from the corresponding author upon request.

\section{Conflicts of Interest}

The authors declare that there are no conflicts of interest regarding the publication of this paper

\section{Acknowledgments}

This work was supported by the National Natural Science Foundation of China (Grants nos. 51409068 and 51209046). This work was also supported by China Postdoctoral Science Foundation (Grant no. 2015M571396).

\section{References}

[1] S. Y. Oakley and D. Nowell, "Prediction of the combined highand low-cycle fatigue performance of gas turbine blades after foreign object damage," International Journal of Fatigue, vol. 29, no. 1, pp. 69-80, 2007.

[2] W. G. Mao and L. Rychlik, "Whipping/springing response in the time series of ship structural stresses," in Conference in LogonnaDaoulas, France, 2012.

[3] W. Mao, J. W. Ringsberg, and I. Rychlik, "The Effect of Whipping/Springing on Fatigue Damage and Extreme Response of Ship Structures," in Proceedings of the ASME 2010 29th International Conference on Ocean, Offshore and Arctic Engineering, pp. 123-131, Shanghai, China.

[4] M. Barhoumi and G. Storhaug, "Assessment of whipping and springing on a large container vessel," International Journal of Naval Architecture and Ocean Engineering, vol. 6, no. 2, pp. 442458, 2014.

[5] C. Dungey and P. Bowen, "The effect of combined cycle fatigue upon the fatigue performance of TI-6AL-4V fan blade material," Journal of Materials Processing Technology, vol. 153154, no. 1-3, pp. 374-379, 2004.

[6] COMMITTEE III.2, "Fatigue and Fracture," in Proceedings of the 16th International ship and offshore structures congress, Southampton, UK, August 2006. 
[7] T.-T. Fu and D. Cebon, "Predicting fatigue lives for bi-modal stress spectral densities," International Journal of Fatigue, vol. 22, no. 1, pp. 11-21, 2000.

[8] I. Lotsberg, "Background for Revision of DNV-RP-C203 Fatigue Analysis of Offshore Steel Structure," in Proceedings of the ASME 2005 24th International Conference on Offshore Mechanics and Arctic Engineering, pp. 297-306, Halkidiki, Greece.

[9] DNV, "Fatigue Design of Offshore Steel Structures," DNV-RPC203, 2012.

[10] W. Huang and T. Moan, "Fatigue Under Combined High and Low Frequency Loads," in Proceedings of the 25th International Conference on Offshore Mechanics and Arctic Engineering, pp. 149-156, Hamburg, Germany.

[11] M. J. KÜhn, Dynamics and design optimization of offshore wind energy conversion systems [Ph.D. thesis], Delft University of Technology, 2001.

[12] C. Han, Y. Ma, X. Qu, M. Yang, and P. Qin, "A practical method for combination of fatigue damage subjected to lowfrequency and high-frequency Gaussian random processes," Applied Ocean Research, vol. 60, pp. 47-60, 2016.

[13] G. Szala and B. Ligaj, "Application of hybrid method in calculation of fatigue life for C45 steel (1045 steel) structural components," International Journal of Fatigue, vol. 91, pp. 3949, 2016.

[14] G. Szala and B. Ligaj, "Description of cyclic properties of steel in variability conditions of mean values and amplitudes of loading cycles," Materials Science Forum, vol. 726, pp. 69-76, 2012.

[15] B. Ligaj and R. Sołtysiak, "Problems of equivalent load amplitude in fatigue life calculations," Polish Maritime Research, vol. 23, no. 1, pp. 85-92, 2016.

[16] G. Jiao and T. Moan, "Probabilistic analysis of fatigue due to Gaussian load processes," Probabilistic Engineering Mechanics, vol. 5, no. 2, pp. 76-83, 1990.

[17] M. A. Miner, "Cumulative damage in fatigue," Journal of Applied Mechanics, vol. 12, pp. A159-A164, 1945.

[18] American Society for Testing and Materials, "ASTM E104985 Standard Practices for Cycle Counting in Fatigue Analysis," ASTM, 1997.

[19] M. Matsuishi and T. Endo, "Fatigue of metals subjected to varying stress," Japan Society of Mechanical Engineers , 1968.

[20] J. Van der Tempel, Design of support structures for offshore wind turbines [Ph.D. thesis], Delft University of Technology, 2006.

[21] American Society for Testing and Materials(ASTM) E8-04 Standard Test Methods for Tension Testing of Metallic Materials. ASTM. 


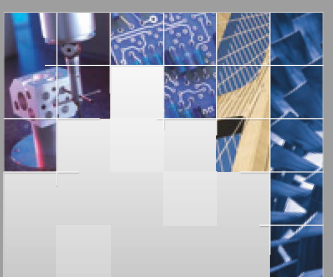

\section{Enfincering}
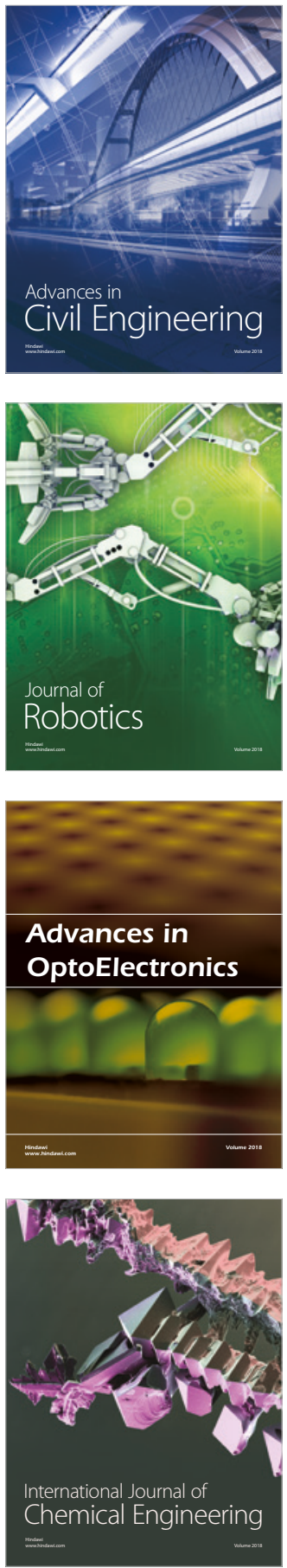

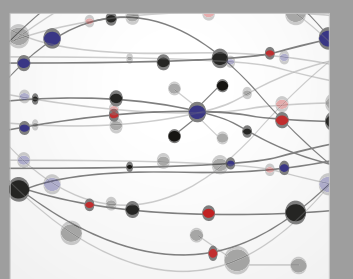

\section{Rotating \\ Machinery}

The Scientific World Journal

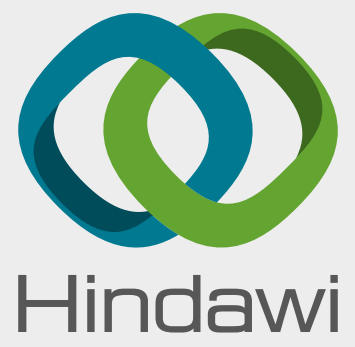

Submit your manuscripts at

www.hindawi.com
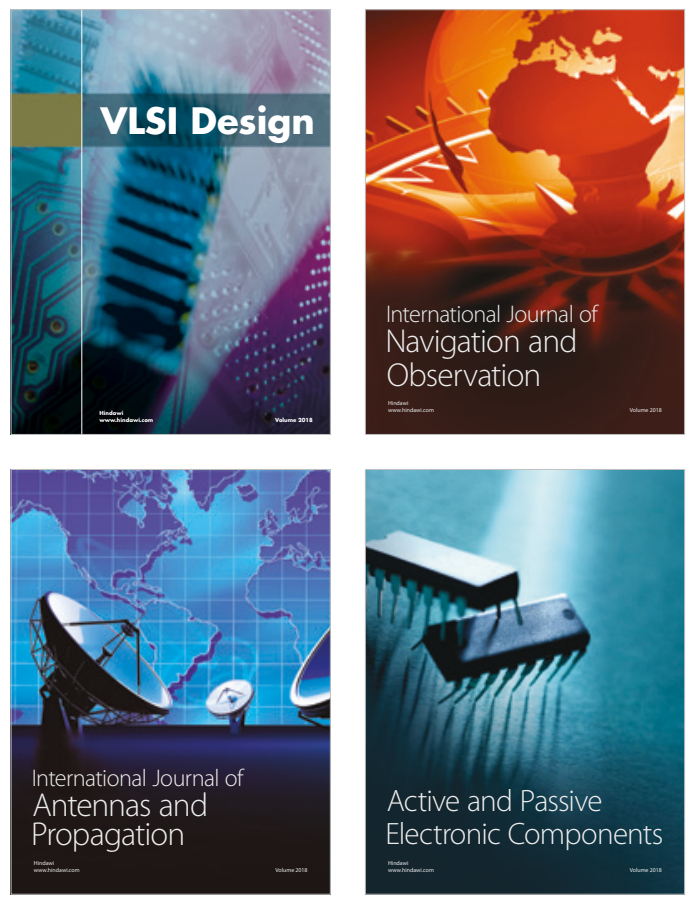
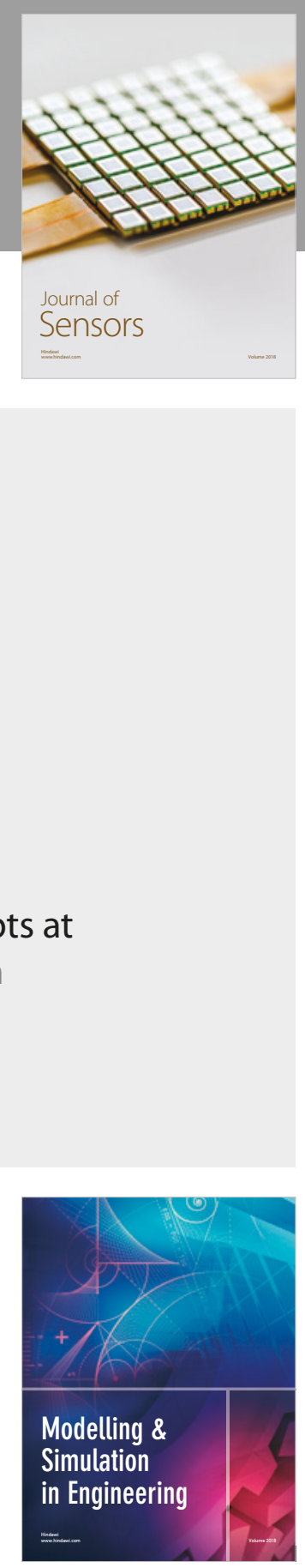

\section{Advances \\ Multimedia}
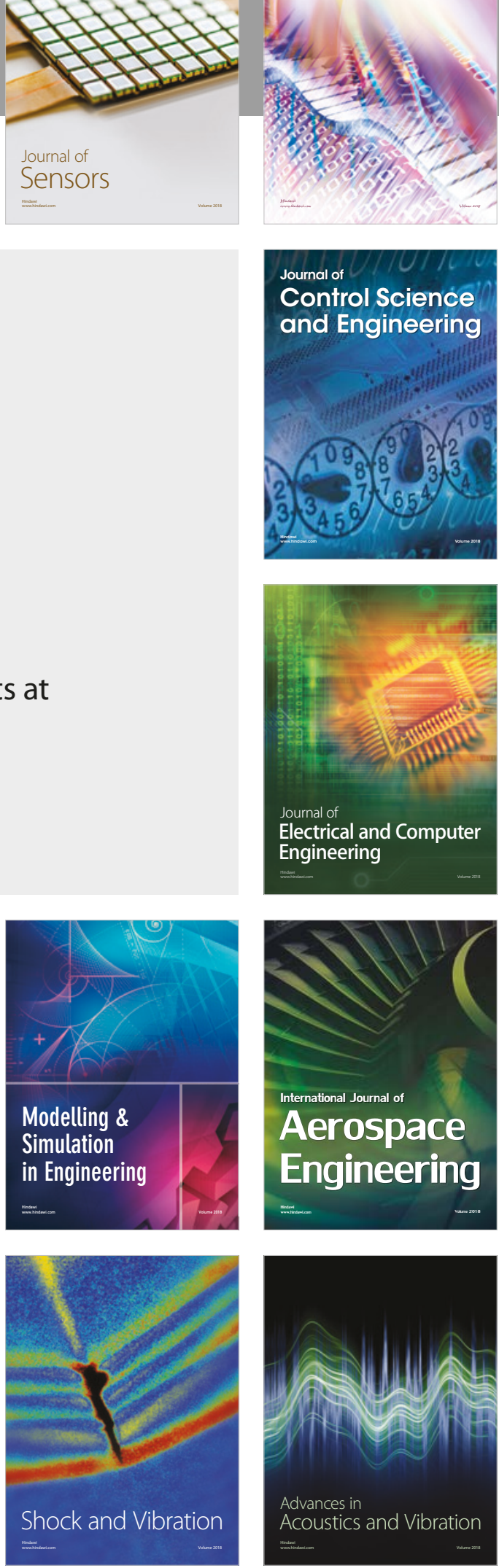